\title{
Denoising of ECG Signals using Multiwavelet Transform
}

\author{
${ }^{* 1}$ S. Yoganand, ${ }^{2}$ B. Madhan Mohan \\ ${ }^{1,2}$ Department of ECE, SV College of Engineering, Tirupati. \\ ${ }^{{ }^{*} \text { Email: } \text { yoganand9@gmail.com }}$
}

Received: 06th October 2017 Accepted: 14th November 2017, Published: 31st December 2017

\begin{abstract}
These days, as the rate of heart ailments expanding progressively, electrocardiogram [ECG] an essential apparatus to analyze the different issues relating to heart. Yet, the recorded ECG frequently contains ancient rarities like electrical cable commotion, gauge clamor, and muscle antiquities. Subsequently denoising of ECG signals is vital for exact analysis of heart ailments. To break down these signs this paper utilizes an intense numerical device called wavelet change. Discrete wavelet transform[DWT] being repetitive and capable, it confronts a couple of issues in the range of correspondence, inorder to stay away from those issues this paper proposes another multiresolution strategy with multi channel called Multiwavelet transform[MWT].
\end{abstract}

Keywords: Multiwavelets, ECG Denoising, Signal to noise ratio, Wavelet methods, lifting wavelet transform.

\section{INTRODUCTION}

In current prescription, there are numerous techniques to analyze coronary illness, for example, electrocardiogram (ECG), ultrasound, attractive reverberation imaging (MRI), and PC tomography (CT). Among these strategies, analysis utilizing electrocardiogram has the benefits of accommodation and minimal effort with the goal that it can be utilized as a part of a wide zone. In any case, certain arrhythmia (a quick, moderate, or unpredictable pulse) which can cause irregular manifestations may happen just sporadically or may happen just under specific conditions, for example, push. Arrhythmia of this sort is hard to acquire on an electrocardiogram following that runs just for a couple of minutes. The ECG is the declaration of difference of bioelectric potential regarding time as the human heart thumps. Because of its usability and noninvasiveness, electrocardiogram assumes an essential part in quiet observing and analysis.

The change in sunlight based movement incorporating electrocardiographic information with varieties in galactic infinite beams, geomagnetic action, and barometrical weight recommends the likelihood of connections among these physical natural varieties and wellbeing dangers, for example, myocardial areas of localized necrosis and ischemic strokes. An expansion in the frequency of myocardial dead tissue in relationship with attractive tempests has been accounted for by Cornélissen et al. [1].

Attractive tempests are found to diminish heart rate inconstancy (HRV) showing a conceivable instrument since a decreased $H R V$ is an imperative factor for coronary supply route infection and myocardial dead tissue. An expansion of 5\% in mortality amid years of maximal sunlight based action is discovered when contrasted and years of negligible sun based movement. These chronodiagnostics are especially imperative for those wandering into areas far from healing facilities.

Goudarzi et al. [2] endeavored to locate the ideal multiwavelet for pressure of ECG signs to be utilized alongside SPIHT codec.

Kania et al. [3] examined the use of wavelet denoising in commotion decrease of multichannel high determination ECG signals. The impact of the choice of wavelet work and the decision of disintegration level on proficiency of denoising process was considered and entire methodology of commotion diminishment were actualized in MATLAB condition utilizing the quick wavelet change. The denoising strategy was observed to be worthwhile since commotion level was diminished in ECG signals, in which clamor decrease by averaging had restricted application, that is, if there should arise an occurrence of arrhythmia.

Helenprabha and Natarajan [4] proposed a strategy utilized for measuring electrical signs produced by fetal heart as measured from multichannel potential chronicles on the mother's body surface. They proposed another class of versatile channel that consolidates the alluring properties of limited motivation reaction (FIR) channel with unbounded drive reaction (IIR) channel. The maternal ECG and fetal signs were mimicked utilizing MATLAB. The gamma channel configuration was actualized in FPGA Spartan 2E which was modified utilizing VHDL. Their outcomes have unraveled the intricate circumstances more dependably than typical versatile techniques utilized before for recouping fetal signs.

Chang et al. [5] proposed measures to influence the ideal channel to plan under various limitations feasible for 
ECG flag handling. Examinations have been directed by them with misleadingly and for all intents and purposes defiled ECG signals for PLI versatile sifting strategy. The appraisals incorporated the union time, the recurrence following effectiveness, the execution time, and the relative insights in time and recurrence area. The outcomes exhibited that there is no general ideal approach for this application up to this point.

Alfaouri and Daqrouq [6] performed wavelet change thresholding strategy for ECG flag denoising. They disintegrated the flag into five levels of wavelet change utilizing the Daubechies wavelet (db4) and decided an edge through a circle to discover the esteem where least mistake was accomplished between the itemized coefficients of limit loud flag and the first flag. The limit esteem was refined tentatively subsequent to utilizing a circle of ascertaining a base mistake between the denoised wavelet subsignals and the first free of clamor subsignals. The test use of the limit result was superior to Donoho's edge especially in ECG flag denoising.

Zhidong and Chan [7] proposed a novel technique for the expulsion of electrical cable recurrence from ECG signals in view of exact mode decay (EMD) and versatile channel. An information driven versatile system called EMD was utilized to break down ECG motion into a progression of characteristic mode capacities (IMFs). The versatile electrical cable cancelation channel was intended to expel the electrical cable obstruction, the reference flag of which was delivered by specific remaking of IMFs. Center ECG signals were utilized to assess the execution of the channel. Results demonstrated that the electrical cable impedance of ECG was evacuated successfully by the new strategy.

Kaur and Singh [8] proposed a mix technique for control line obstruction lessening in ECG. The strategies were moving normal strategy and utilizing the IIR score attributes. Their outcomes indicated diminishment in the electrical cable commotion in the ECG flag utilizing the proposed channel that has less coefficients and thus lesser calculation time for constant preparing.

Haque et al. [9] utilized wavelet technique to recognize the little varieties of ECG highlights. They mimicked standard ECG motions and in addition the reenacted commotion debased flag utilizing FFT and wavelet for appropriate element extraction. They observed wavelet to be better than the traditional FFT technique in finding the little variations from the norm in electrocardiogram signals.

Tan and Lei [10] utilized wavelet change to sift through clamor obstructions of electrocardiogram signals for the separating of the myoelectric impedance, the power recurrence impedance, and the benchmark float. Right off the bat Coif4 wavelet was adjusted to deteriorate electrocardiogram signals containing commotions. Furthermore, the delicate and hard edge esteem measured high-recurrence coefficients of each scale lastly the electrocardiogram were recreated utilizing highrecurrence coefficients of each scale which were evaluated by the edge esteem. Investigations demonstrated that wavelet change had great constant separating impact and it had a bigger number of points of interest than customary techniques.

This paper proposes MWT for denoising of ECG signals and this strategy is named as proposed calculation, denoising of ECG signals utilizing DWT and lifting wavelet change (LWT) are dealt with as the current strategies.

\section{Multiwavelet Method}

Multiwavelets is another subordinate of wavelet hypothesis as of late. As of late, much intrigue has been produced in the investigation of the multiwavelets where more than one scaling capacities and mother wavelet are utilized to speak to a given flag. The principal development for polynomial multiwavelets was given by Albert, who utilized them as a reason for the portrayal of specific administrators. Afterward, Geronimo, Hardin, and Massopust developed a multiscaling capacity with 2 parts utilizing fractal insertion.

Disregarding numerous hypothetical outcomes on multiwavelet, their effective applications to different issues in flag preparing are as yet constrained. Not at all like scalar wavelets in which Mallet's pyramid calculation have given an answer for good flag decay and reproduction, a great system for the utilization of the multiwavelet is as yet not accessible. All things considered, a few scientists have proposed strategy for how to apply a given multiwavelet channel to flag and picture decay.

\subsection{Multiscaling Functions and Multiwavelets}

The concept of multiresolution analysis can be extended from the scalar case to general dimension $r \mathrm{~N}$. A vector valued function $\varphi=\left[\varphi_{1}, \varphi_{2}, \ldots \ldots \ldots . \varphi_{r}\right]^{T}$ belonging to $L^{2}(R)^{r}$ and $r \mathrm{~N}$ is called a multiscaling function if the sequence of closed spaces

$$
V_{j}=\operatorname{span}\left\{2^{j / 2} \varphi_{i}\left(2^{j}-k\right): 1 \leq i \leq r, k \in Z\right\} .
$$

$j \in Z$ constitute a multiresolution analysis (MRA) of multiplicity $r$ for $L^{2}(R)$. The multiscaling function must satisfy the two-scale dilation equation

$$
\varphi(t)=\sqrt{2} \sum_{k} G_{k} \varphi(2 t-k)
$$


Now let $W_{j}$ denote a complementary space of $V_{j}$ in $V_{j+1}$. The vector valued function $\Psi=\left[\Psi_{1}, \Psi_{2}, \ldots \ldots . \Psi_{r}\right]^{T}$ such that

$$
W_{j}=\operatorname{span}\left\{2^{j / 2} \varphi_{i}\left(2^{j}-k\right): 1 \leq i \leq r, k \in Z\right\} .
$$

$j \in Z$ is called a multiwavelet. The multiscaling function must satisfy the two-scale equation

$$
\Psi(t)=\sqrt{2} \sum_{k} H_{k} \varphi(2 t-k)
$$

$H_{k} \in L^{2}(Z)^{r \times r}$ is an $r \times r$ matrix of coefficients. The two-scale equations [2] and[4] can be realized as a multifilter bank operating on input data streams and filtering them in two $2 \mathrm{r}$ output data streams, each of which is downsampled by a factor of two. If $x(t)$ is the given signal and it is assumed that $x(t) \in V_{o}$, then

$$
x(t)=\sqrt{2} \sum_{k} V_{0, k}^{T} \varphi(t-k)
$$

And the scaling coefficient $V_{1, k}^{T}$ of the first level can be considered as a result of low-pass multifiltering and downsampling as follows:

$$
V_{1, k}=\sum_{m} G_{m-2 k} V_{0, m}
$$

Analogously, the first level multiwavelet coefficients are obtained using high-pass multifiltering and downsampling as follows:

$$
W_{1, k}=\sum_{m} H_{m-2 k} V_{0, m}
$$

Full multiwavelet decomposition of the signal $x(t)$ can be found by iterative filtering of the scaling coefficient as follows:

$$
\begin{aligned}
V_{j, k} & =\sum_{m} G_{m-2 k} V_{j-1, m} \\
W_{j, k} & =\sum_{m} H_{m-2 k} V_{j-1, m}
\end{aligned}
$$

Note that $V_{j, k}$ and $W_{j, k}$ are $r \times 1$ column vectors.

\subsection{Advantages of Wavelets and Multiwavelets} Compared to Conventional Filtering Techniques

(i)The Fourier change neglects to break down the nonstationary flag, though wavelet change permits the segments of a nonstationary flag to be examined.

(ii)Wavelet changes holds the property of multiresolution to give both time and recurrence space data in a synchronous way.

(iii)A set of wavelets which are integral can deteriorate the given information without holes or cover so the disintegration procedure turns out to be numerically reversible.

\subsection{Comparison between Wavelet and Multiwavelet}

Multiwavelets contain numerous scaling capacities, while scalar wavelets contain one scaling capacity and one wavelet. This prompts more degrees of flexibility in developing wavelets. In this manner, contradicted to scalar wavelets, properties, for example, conservative help, orthogonality, symmetry, vanishing minutes, and short help can be assembled at the same time in multiwavelets which are major in flag handling.

The expansion in level of flexibility in multiwavelets is gotten to the detriment of supplanting scalars with frameworks, scalar capacities with vector capacities, and single networks with piece of lattices. Be that as it may, prefiltering is a basic assignment which ought to be performed for any utilization of multiwavelet in flag preparing.

\section{RESUlTS}

In order to test the proposed algorithm, 4 ECG signals from MIT-BIH Arrhythmia Database were considered [113.dat, 119.dat, 208.dat, 214.dat] with different rate of variations in the signal. These four ECG signals were tested with both existing and proposed algorithms, by varying the input signal to noise ratio from $-10 \mathrm{~dB}$ to $10 \mathrm{~dB}$.

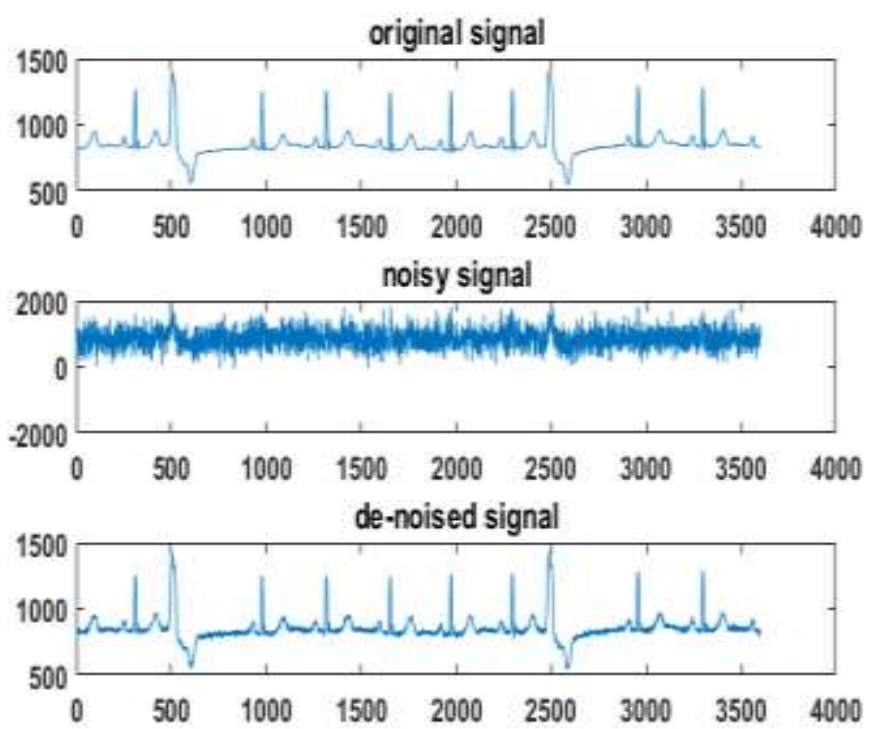

Fig.1. Denoising using lifting wavelets (LWT).

Denoising the signal using DWT fails, when the rate of variations is high, it works better and produces better signal to noise ratio for mere sparse signal and fails to attain the same performance when data rate is high.

Denoising the signal using LWT is producing better results compared to DWT but fails to process low frequency signals and from the comparison table given below it will be clear that the mean square error generated by this method is large compared to DWT and proposed method. 
Proposed method, i.e., denoising of signals using multiwavelets produces better results in terms of both signal to noise ratio and mean square error. Same can be understood from the given below table and graphical representations.

\begin{tabular}{|c|c|c|c|}
\hline Method & Signal Name & PSNR[dB] & MSE \\
\hline \multirow{4}{*}{ DWT } & 113. DAT & 35.7765 & 193.1501 \\
\cline { 2 - 4 } & 119. DAT & 31.9459 & 150.7813 \\
\cline { 2 - 4 } & 208. DAT & 31.5486 & 203.9454 \\
\cline { 2 - 4 } & 214. DAT & 32.719 & 192.9849 \\
\hline \multirow{4}{*}{ LWT } & 113. DAT & 28.6125 & 193.3602 \\
\cline { 2 - 4 } & 119. DAT & 32.4084 & 147.1837 \\
\cline { 2 - 4 } & 208. DAT & 31.6707 & 202.3619 \\
\cline { 2 - 4 } MWT & 214. DAT & 36.851 & 203.2513 \\
\hline \multirow{4}{*}{} & 113. DAT & 31.8811 & 192.7762 \\
\cline { 2 - 4 } & 119. DAT & 31.134 & 148.5508 \\
\cline { 2 - 4 } & 208. DAT & 33.6627 & 192.496 \\
\cline { 2 - 4 } & 214. DAT & 35.3067 & 201.0282 \\
\hline
\end{tabular}

Table1: Comparison of SNR and MSE

From the above comparison table it is clear that proposed method that is Multiwavelet transform is producing better and constant results irrespective of data rate even at low input SNR( i.e., $-10 \mathrm{~dB}$ ).
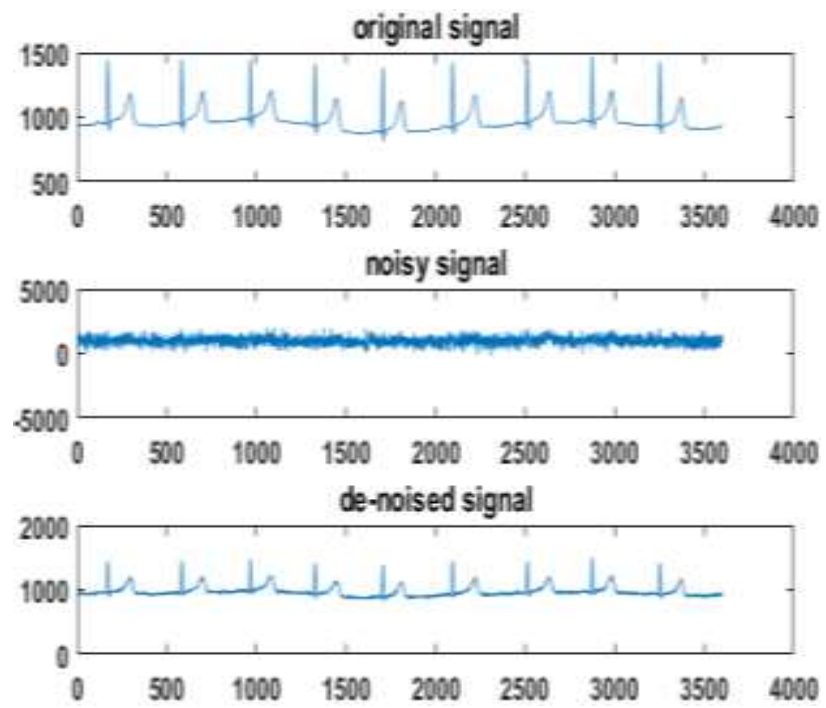

Fig. 2. Denoising using the proposed method

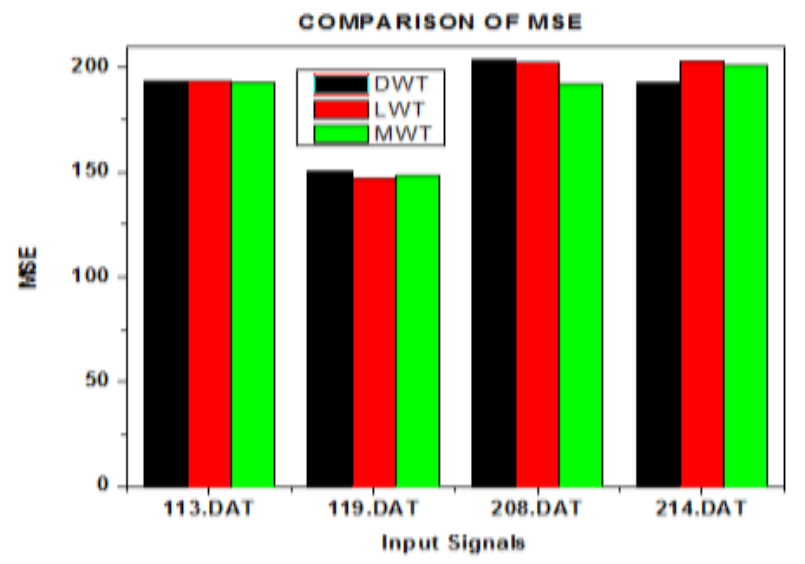

Fig. 3. Comparison of Mean Square Error (MSE).

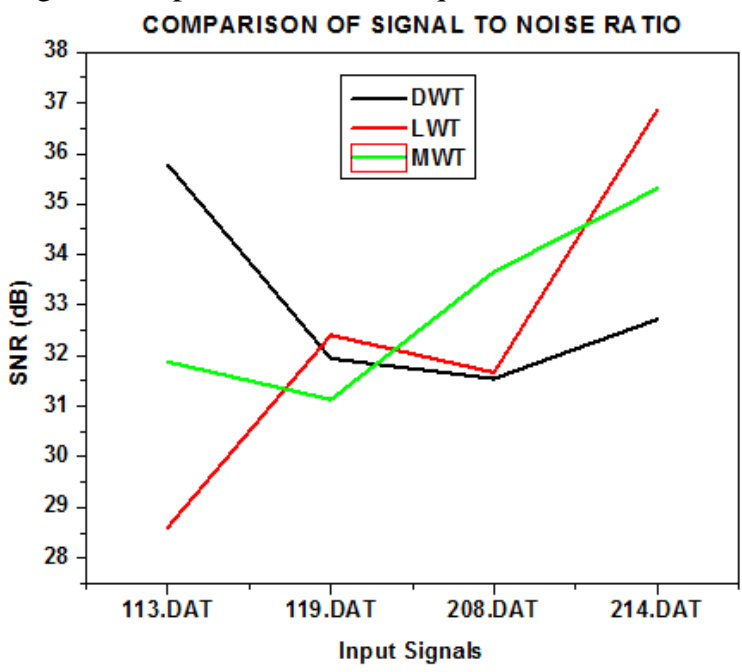

Fig. 4. Comparison of Signal to Noise Ratio (SNR)

\section{CONCLUSION}

Discrete Wavelet Transform (DWT), being redundant and powerful, it fails to process the signal with fast heart beat; it does well with slow heart beat ECG signals. On the other hand the proposed method i.e., MWT outperforms both the existing methods like DWT and LWT in terms of SNR and MSE respectively, LWT even though, it produces better signal power but it fails to retain the original ECG as it is and it was evaluated in terms of MSE.

MWT can perform even better and produces better results if a proper thresholding is applied to the wavelet coefficients.

\section{REFERENCES}

1.C. Germaine, F. Halberg, T. Breus et al., "Non-photic solar associations of heart rate variability and myocardial infarction," Journal of Atmospheric and Solar-Terrestrial Physics, vol. 64, no. 5, pp. 707-720, 2002. 
2.M. M. Goudarzi, A. Taheri, M. Pooyan, and R. Mahboobi, "Multiwavelet and biological signal processing," International Journal of Information Technology, vol. 24, pp. 264-272, 2006.

3. M. Kania, M. Fereniec, and R. Maniewski, "Wavelet denoising for multi-lead high resolution ECG signals," Measurement Science Review, vol. 7, Section 2, no. 4, pp. 30-33, 2007.

4. K. Helenprabha and A. M. Natarajan, "FPGA implementation of gamma filter for extracting fetal electrocardiogram," IETE Journal of Research, no. 53, pp. 433-440, 2007.

5. F. C. Chang, C. K. Chang, K. Y. Chi, and Y. D. Lin, "Evaluation measures for adaptive PLI filters in ECG signal processing," Computers in Cardiology, vol. 34, pp. 529-532, 2007.

6. M. Alfaouri and K. Daqrouq, "ECG Signal Denoising by wavelet transform thresholding," The American Journal of Applied Sciences, vol. 53, pp. 276-281, 2008.

7. Z. Zhidong and M. Chan, "A novel cancellation method of powerline interference in ECG signal based on EMD and adaptive filter," in Proceedings of the 11th IEEE International Conference on Communication Technology, pp. 517-520, 2008.

8. M. Kaur and B. Singh, "Powerline interference reduction in ECG using combination of MA method and IIR notch," International Journal of Recent Trends in Engineering, vol. 2, no. 6, pp. 125-129, 2009.

9. A. K. M. F. Haque, M. H. Ali, M. A. Kiber, and M. T. Hasan, "Detection of small variations of ECG features using wavelet," ARPN Journal of Engineering and Applied Sciences, vol. 4, no. 6, pp. 27-30, 2009.

10. Y.-F. Tan and D. Lei, "Study on Wavelet Transform in the processing for ECG signals," in Proceedings of the WRI World Congress on Software Engineering (WCSE '09), vol. 9, pp. 515-518, IEEE Computer Society, May 2009. 